\title{
O PAPEL DO PEDIATRA NO DIAGNÓSTICO PRECOCE DO RETINOBLASTOMA
}

\author{
Célia B. Gianotti Antoneli*, flávio Steinhorst, Karina de Cássia Braga Ribeiro, \\ Martha M. M. Chojniak, Paulo Eduardo RS Novaes, Victor Arias, Alois Bianchi \\ Trabalho realizado no Centro de Tratamento e Pesquisa do Hospital do Câncer AC Camargo, São Paulo, SP
}

RESUMO - OBJETIVO. 0 retinoblastoma é o tumor intra-ocular mais freqüente na infância. A forma de apresentação mais comum é leucocoria, seguida pelo estrabismo. Nos países em desenvolvimento, pela demora diagnóstica e maior tempo de encaminhamento, encontramos um grande número de pacientes portadores de tumores extra-oculares.

Métodos. Duzentos e trinta e nove pacientes com retinoblastoma admitidos nos departamentos de Pediatria e Oftalmologia do Hospital do Câncer AC Camargo, no período de 1986 a 1995, foram avaliados quanto ao tempo de encaminhamento e extensão do tumor. A análise estatística incluiu o cálculo de frequêencias absolutas e relativas, bem como o teste exato de Fisher, para a verificação da associação entre variáveis categorias.

Resultados. Pacientes que foram encaminhados em um período menor que 6 meses apresentaram maior freqüência de doença intra-ocular quando comparados com pacientes com tempo de encaminhamento maior que seis meses $(75 \%$ vs. $25 \%$ $\mathrm{p}<0,001)$. Não houve diferença estatisticamente significativa para tumores extra-oculares.

Conclusöes. 0 diagnóstico precoce é considerado o ponto mais importante no tratamento de pacientes com retinoblastoma. Os pediatras são capazes de detectar, em uma consulta simples de ambulatório, os sinais e sintomas desta doença e devem ter sempre em mente o diagnóstico de retinoblastoma, possibilitando o encaminhamento precoce a centros especializados.

Unitermos: Retinoblastoma. Sinais. Sintomas. Diagnóstico.

\section{INTRODUÇÃO}

Embora a ocorrência do retinoblastoma seja rara, acometendo I// 5.000 a I/20.000 nascidos vivos', o pediatra geral tem um papel fundamental no diagnóstico dos tumores oculares, pois pode reconhecer um problema ocular que não tenha sido notado pelos pais. Algumas vezes os pais percebem o "brilho" diferente no olho da criança e a queixa é subestimada pelo pediatra. Setenta e cinco por cento dos casos ocorrem nos três primeiros anos de vida. $O$ sinal mais freqüente é a leucocoria (reflexo do olho de gato), seguido do estrabismo ${ }^{2,3}$. Leucocoria é definida como um reflexo anormal da pupila à luz.

Com o conhecimento dos sinais e sintomas dos tumores oculares, cabe ao pediatra 0 encaminhamento ao oftalmologista, que tomará as medidas cabíveis frente ao caso. O diagnóstico precoce e o reconhecimento de fatores de risco para metástases traçam o esquema terapêutico apropriado para cada situação que se apresente.

*Correspondência: Hospital do Câncer A C Camargo. Centro de Tratamento e Pesquisa Rua Prof. Antonio Prudente, 211 Liberdade - São Paulo - SP

CEP: 01509-900 - Fone: (11) 3272-5166 E-mail: cantoneli@terra.com.br
Quanto mais precoce o diagnóstico, maiores são as chances de preservação da visão e maiores as taxas de sobrevida.

\section{Pacientes e métodos}

Pacientes portadores de retinoblastoma admitidos no período de 1986 e 1995 nos Departamentos de Pediatria e Oftalmologia do Hospital do Câncer foram avaliados quanto ao tempo de encaminhamento e extensão do tumor.

$\mathrm{Na}$ análise estatística, consideramos dois períodos de estudo, sendo o primeiro entre os anos de 1986 a 1990, quando 105 pacientes foram incluídos no estudo e o segundo, entre os anos de 199| e 1995, composto por I52 pacientes.

O tempo de encaminhamento foi definido como o período compreendido entre a data do aparecimento do primeiro sinal e/ou sintoma e a data da chegada ao hospital. A extensão da doença foi classificada segundo o estadiamento de Reese-Ellsworth (tumores intraoculares) $)^{4}$ ou CCG (tumores extra-oculares) ${ }^{5}$. A análise estatística incluiu freqüências absolutas e relativas e o teste exato de Fisher para verificação da associação entre as variáveis categóricas, com um nível de significância estatística igual a 5\%.

\section{Resultados}

Entre os 257 pacientes da amostra, observamos que 198 (77\%) eram portadores de tumores intra-oculares e, destes, $187(94,4 \%)$ foram admitidos com doença classificada como Reese-Ellsworth estádio $\mathrm{V}^{6}$.

No primeiro período, o tempo médio de encaminhamento foi 7,5 meses, com um desvio padrão de 7,79. No segundo período, o tempo médio de encaminhamento foi 5,3 meses, com desvio padrão de 6,84.

Houve associação estatisticamente significativa entre tempo de encaminhamento e a extensão do tumor $(p<0,00 \mathrm{I})$. A Tabela I mostra o tempo de encaminhamento nos dois períodos e a Tabela 2 mostra a relação entre a extensão da doença e o tempo de encaminhamento nos dois períodos.

Quando comparamos o tempo de encaminhamento para pacientes com tumores intra-oculares, observamos que houve uma diferença estatisticamente significativa $(p=0,00 I)$, embora essa diferença não tenha sido observada para pacientes com tumores extra-oculares $(p=0,977)$, conforme podemos observar na Tabela 3. 
Tabela I - Número e porcentagem de pacientes, segundo período de admissão e tempo de encaminhamento, Centro de Tratamento e Pesquisa Hospital do Câncer AC Camargo, 1986-1995

\begin{tabular}{|c|c|c|c|}
\hline \multirow[t]{2}{*}{ Período } & \multicolumn{2}{|c|}{ Tempo de encaminhamento (\%) } & \multirow[t]{2}{*}{$\mathrm{p}^{*}$} \\
\hline & $\leq 6$ meses & $>6$ meses & \\
\hline $\begin{array}{l}\text { I } \\
\text { II } \\
\text { Total }\end{array}$ & $\begin{array}{l}62(59) \\
100(75) \\
162(68)\end{array}$ & $\begin{array}{l}43(41) \\
34(25) \\
77(32)\end{array}$ & 0,011 \\
\hline
\end{tabular}

* nivel de significância estatística segundo o teste exato de Fisher

Tabela 2 - Número e porcentagem de pacientes, segundo período de admissão e extensão do tumor, Centro de Tratamento e Pesquisa Hospital do Câncer AC Camargo, 1986-1995

\begin{tabular}{lccc}
\hline Extensão & \multicolumn{2}{c}{ Tempo de encaminhamento(\%) } & p* \\
\cline { 2 - 3 } & $\mathbf{S 6 \text { meses }}$ & $>6$ meses & \\
Intra-ocular & $\mid 37(74,9)$ & $46(25,1)$ & $<0,00 \mid$ \\
Extra-ocular & $25(44,6)$ & $3 \mid(55,4)$ & \\
Total & $\mid 62(68,0)$ & $77(32,0)$ & \\
\hline
\end{tabular}

* nivel de significância estatística segundo 0 teste exato de Fisher

\begin{tabular}{|c|c|c|c|c|c|c|}
\hline \multirow[t]{3}{*}{ Período } & \multicolumn{6}{|c|}{ Tempo de encaminhamento (\%) } \\
\hline & \multicolumn{3}{|c|}{ Intra-oculares } & \multicolumn{3}{|c|}{ Extra-oculares } \\
\hline & $\leq 6$ meses & $>6$ meses & $\mathrm{p}^{*}$ & $\leq 6$ meses & $>6$ meses & $p^{*}$ \\
\hline । & $50(65,0)$ & $28(36,0)$ & 0,001 & $12(44,4)$ & $\mid 5(55,6)$ & 0,977 \\
\hline$\|$ & $87(83,0)$ & $18(17,0)$ & & $13(44,7)$ & $16(55,2)$ & \\
\hline Total & $137(75,0)$ & $46(25,0)$ & & $25(44,7)$ & $31(55,3)$ & \\
\hline
\end{tabular}

* nível de significância estatística segundo o teste exato de Fisher

\section{Discussão}

O Hospital do Câncer AC Camargo é um centro de referência para tratamento de tumores oculares. De acordo com o Registro Hospitalar de Câncer Pediátrico da instituição, o retinoblastoma representa 1 I, I\% de todos os tumores pediátricos?. No ano de 1988, foram admitidos 18 pacientes com tumores unilaterais e nove com tumores bilaterais. Quando comparada com o ano de 1994, a proporção de pacientes com tumores unilaterais se manteve praticamente a mesma (I2 casos), enquanto que a proporção de pacientes com tumores bilaterais duplicou (19 pacientes). Esse fato se explica pela aquisição das placas de cobalto, responsável por maiores taxas de preservação de visão.

Em um estudo do perfil dos pacientes admitidos entre os anos de 1975 e 1985 , observou-se que um grande número de pacientes era admitido com tumores extra- oculares ao diagnóstico ${ }^{8}$. No período compreendido entre os anos de 1975 e 1985, I 53 pacientes portadores de retinoblastoma foram admitidos nos Departamentos de Pediatria e Oftalmologia do Hospital do Câncer AC Camargo. Desses, 43,8\% apresentavam tumor intra-ocular e 56,2\%, extra-ocular. Nesse estudo concluiu-se que o risco de tumor extra-ocular era nove vezes maior quando o tempo de encaminhamento era maior que seis meses. Entre os anos de 1986 e 1990, foram admitidos 105 pacientes com retinoblastoma. A proporção de pacientes com tumor intra e extra-ocular foi respectivamente $75,2 \%$ e $24,8 \%$, fato este que se repetiu entre 120 pacientes avaliados no período compreendido entre os anos de 199| e 1994 (tumores intraoculares: $75,8 \%$ e tumores extra-oculares: 24,2\%). Neste último período estudado, 47,5\% dos pacientes apresentaram um tempo de encaminhamento menor que três meses?.
O aumento da taxa de pacientes com tumores intra-oculares deveu-se a campanhas de esclarecimento à população leiga através de artigos em revistas não médicas e out-doors colocados em pontos estratégicos da cidade. A população médica recebeu informação através de cursos ministrados em Faculdades de Medicina e através de publicação em revista médica.

Infelizmente, apesar do tempo de encaminhamento ter diminuído e do diagnóstico de tumor intra-ocular ter aumentado, ainda recebemos pacientes com tumores intraoculares avançados, sem possibilidade de preservação da visão, o que impede que esses pacientes recebam terapêutica conservadora, fazendo com que sejam submetidos à enucleação.

Apesar de todos os esforços, um estudo de Rodrigues e Camargo, relativo a uma coorte de 2081 pacientes admitidos no Departamento de Pediatria do Hospital do Câncer AC Camargo, no período de janeiro de 199| a abril de 2002, descreveu 327 pacientes com retinoblastoma $(18,1 \%)$, com tempo médio de encaminhamento de 5,8 meses. Neste estudo, 269 pacientes apresentavam doença localizada, 42 doença avançada e 16 metástases a distância ${ }^{10}$.

Esse fato ocorre em países latino-americanos como Chile, Peru e Argentina. Dados publicados por Chantada et al. apontaram que, entre 95 pacientes, a consulta demorou mais de 24 semanas em 20 deles". Conclusões interessantes desse estudo apontam para o fato de que os pacientes com seguro saúde apresentavam uma freqüência maior de tumores intra-oculares quando comparados a pacientes sem seguro saúde: 33/39 versus 28/56 ( $p<0,001)$. Outra associação interessante foi aquela encontrada entre a escolaridade dos pais e a extensão da doença. Entre os pais com educação superior, 28 pacientes apresentavam tumor intra-ocular e cinco, extra-ocular, enquanto que, no grupo de pais com educação primária e inferior, esses números foram respectivamente 33 e $29(p=0,005)$.

Dados de outros países como México mostram que o tempo de encaminhamento em um estudo de 4940 pacientes foi de cinco meses e os fatores que contribuíram para esse fato foram: baixo nível educacional, não acesso a seguro social e residência fora da capital ${ }^{\prime 2}$. 
Butros et al..$^{13}$ revelaram que os pacientes recebidos no Memorial Sloan - Kettering Cancer Center, em Nova York, apresentaram um tempo de encaminhamento para o sintoma de leucocoria de 1,5 mês e de dois meses para o sintoma estrabismo. Esse trabalho revela que $30 \%$ dos médicos foram responsáveis pelo diagnóstico tardio. Dados da África do Sul, publicados por Strahendorf ${ }^{14}$, demonstraram que a incidência de retinoblastoma é de 15,6\%. Doença metastática foi diagnosticada em 32\% das crianças atendidas e a sobrevida global foi $44 \%$.

O atraso evitável é uma conseqüência do desconhecimento médico e leigo, aliado ao medo do diagnóstico para ambos.

\section{Conclusão}

Cabe ao pediatra geral o diagnóstico precoce do retinoblastoma. Campanhas para a população leiga e médica são de fundamental importância. $O$ ideal seria a detecção da doença em estágios assintomáticos, mas os programas de rastreamento são impraticáveis e caros.

Com o diagnóstico precoce do retinoblastoma, elevam-se as taxas de cura e se consegue preservar a visão em um grande número de pacientes, minimizando o tratamento e maximizando a qualidade de vida. Cabe a cada um de nós reintegrar esse paciente à sociedade de forma não discriminatória para que exerça sua cidadania de maneira plena como seus pares.

\section{Agradecimentos}

Os autores agradecem o apoio da Fundação de Amparo à Pesquisa do Estado de São Paulo (FAPESP - sob número 0 I/ 0829-5), para a realização deste trabalho.

Conflito de interesse: não há.

\section{SUMMARY}

The Pediatrician's ability to RECOGNIZE THE PRESENTING SIGNS AND SYMPTOMS OF RETINOBLASTOMA.

BACKGROUND. Retinoblastoma is the most frequent primary intraocular tumor in children. The mostcommon presenting sign is leukocoria and the second one is strabism. In the developing countries extra ocular disease is seen more frequently because of delayed referral.

MetHOdS. Two hundred and thirty-nine patients with retinoblastoma admitted at the Pediatrics and Ophthalmology Departments of the Hospital do Câncer AC Camargo from 1986 to 1995 were evaluated regarding lag-time and tumorextension.

RESULTS. Patients referred within less than 6 months presented a higher rate of intraocular disease compared with patients having a lagtime greater than 6 months (75\% vs. 25\%, $p<0.001$ ). No statistically significant difference was noted for patients with extra-ocular tumors.

Conclusion. Early diagnosis is the gold standard for patients with Retinoblastoma and the pediatrician's ability to recognize the presenting signs and symptoms can lead to early diagnosis and efficient treatment. [Rev Assoc Med Bras 2004; 50(4): 400-2]

KEY wORDS: Retinoblastoma. Symptoms and diagnosis.

\section{REFERÊNCIAS}

I. Tamboli A, Podgor MJ, Horm JW. The incidence of retinoblastoma in the United States: 1974 through 1985. Arch Ophthalmol 1990; 188: 128-32. [abstract].

2. Abramson DH, Servodidio CA. Retinoblastoma. Optom Clin 1993; 3:49-61.

3. Shields CL, Shields JA Review recent developments in the management of retinoblastoma J Pediatr Ophthalmol Strabismus 1999; 36:8-18
4. Reese AB, Ellsworth RM. Management of retinoblastoma. Ann NY Acad Sci 1964; | | 4:958-62.

5. Wolff JA, Boesel C, Ellsworth R. Extraocular retinoblastoma. New York; 1978. (Children Cancer Study Group- Protocol CCSG 962).

6. Antoneli CBG, Erwenne C, De Camargo B, Bianchi A. Retinoblastoma in a developing country: the challenge of early diagnosis. Med Pediatr Oncol 1977; 29:373-SL2. [abstract].

7. Ribeiro KCB, de Camargo B, Torloni $\mathrm{H}$. Registro hospitalar de câncer pediátrico. [monografia]. São Paulo: Fundação Antonio Prudente; 1999.

8. Erwenne CM, Franco EL Age and lateness of referral as determinants of extraocular Retinoblastoma. Opthalmic Pediatr Genet 1989; 10:179-84.

9. Antoneli CBG Retinoblastoma: análise da evolução clínica de portadores de retinoblastoma submetidos a tratamento multidisciplinar [tese]. São Paulo: Faculdade de Medicina, Universidade de São Paulo; 1999

10. Rodrigues KE, De Camargo B. Diagnóstico precoce do câncer infantil: responsabilidade de todos. Rev Assoc Med Bras 2003; 49:29-34.

II. Chantada G, Fandiño A, Manziti J, Urrutia L, Schvartzman E Late diagnosis of Retinoblastoma in a developing country. Arch. Dis. Child 1999; 80: 17I-4.

12. Fajardo-Gutierrez A, Sandoval-Mex AM, Mejia-Arangure JM, Rencon-Macias ME, Martinez-Garcia MC. Clinical and social factors that affect the time to diagnosis of mexican children with cancer Med Pediatr Oncol 2002; 39:25-31.

13. ButrosLJ, Abramson DH, Dunkel IJ Delayed diagnosis of retinoblastoma: analysis of degree, cause, and potential consequences. Pediatrics 2002; 109:EA45

14. Strahendorf $C$. Relative frequency and treatment of Retinoblastoma in Johannesburg, South Africa: a new vision for developing countries. Med Pediatr Oncol 1997; 29: 373SL-3. [abstract].

Artigo recebido: 19/09/03 Aceito para publicação: 12/1 //03 